\title{
Diffusion enhancement in core-softened fluid confined in nanotubes
}

Cite as: J. Chem. Phys. 137, 084504 (2012); https://doi.org/10.1063/1.4746748

Submitted: 15 March 2012 . Accepted: 03 August 2012. Published Online: 23 August 2012

J. R. Bordin, A. B. de Oliveira, A. Diehl, and Marcia C. Barbosa

ARTICLES YOU MAY BE INTERESTED IN

Enhanced flow of core-softened fluids through narrow nanotubes

The Journal of Chemical Physics 140, 194504 (2014); https://doi.org/10.1063/1.4876555

How fast does water flow in carbon nanotubes?

The Journal of Chemical Physics 138, 094701 (2013); https://doi.org/10.1063/1.4793396

A general purpose model for the condensed phases of water: TIP4P/2005

The Journal of Chemical Physics 123, 234505 (2005); https://doi.org/10.1063/1.2121687

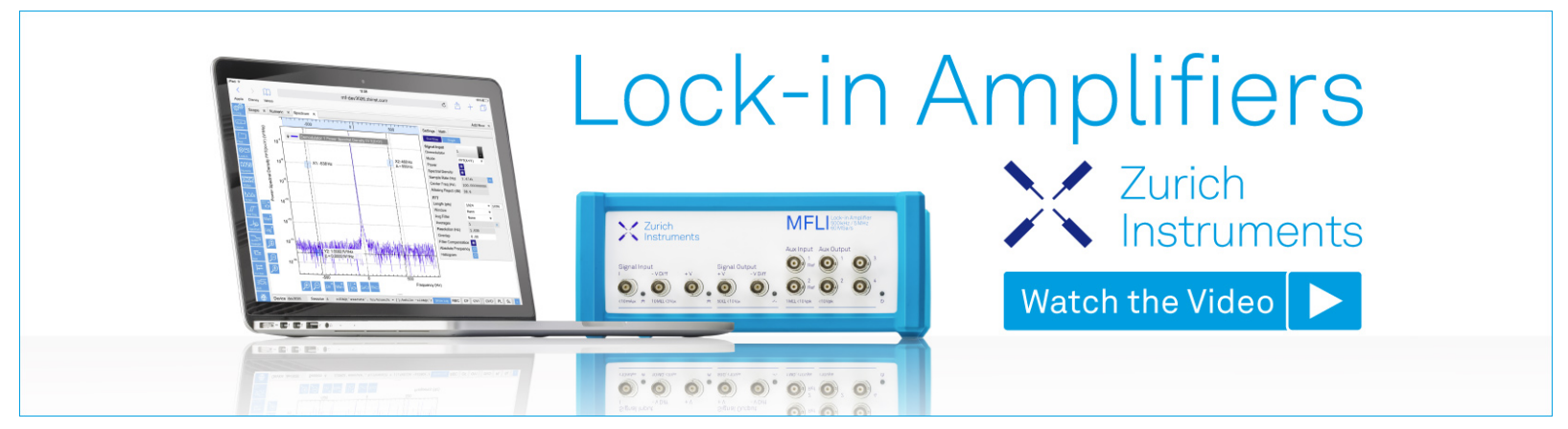

J. Chem. Phys. 137, 084504 (2012); https://doi.org/10.1063/1.4746748

137, 084504

(c) 2012 American Institute of Physics. 


\title{
Diffusion enhancement in core-softened fluid confined in nanotubes
}

\author{
J. R. Bordin, ${ }^{1, a)}$ A. B. de Oliveira, ${ }^{2, b)}$ A. Diehl, ${ }^{3, c)}$ and Marcia C. Barbosa ${ }^{4, d)}$ \\ ${ }^{1}$ Programa de Pós-Graduação em Física, Instituto de Física, Universidade Federal do Rio Grande do Sul, \\ Caixa Postal 15051, CEP 91501-970 Porto Alegre, RS, Brazil \\ ${ }^{2}$ Departamento de Física, Universidade Federal de Ouro Preto, Ouro Preto, MG 35400-000, Brazil \\ ${ }^{3}$ Departamento de Física, Instituto de Física e Matemática, Universidade Federal de Pelotas, \\ Caixa Postal 354, CEP 96010-900 Pelotas, RS, Brazil \\ ${ }^{4}$ Instituto de Física, Universidade Federal do Rio Grande do Sul, Caixa Postal 15051, \\ CEP 91501-970 Porto Alegre, RS, Brazil
}

(Received 15 March 2012; accepted 3 August 2012; published online 23 August 2012)

\begin{abstract}
We study the effect of confinement in the dynamical behavior of a core-softened fluid. The fluid is modeled as a two length scales potential. This potential in the bulk reproduces the anomalous behavior observed in the density and in the diffusion of liquid water. A series of $N p T$ molecular dynamics simulations for this two length scales fluid confined in a nanotube were performed. We obtain that the diffusion coefficient increases with the increase of the nanotube radius for wide channels as expected for normal fluids. However, for narrow channels, the confinement shows an enhancement in the diffusion coefficient when the nanotube radius decreases. This behavior, observed for water, is explained in the framework of the two length scales potential. (C) 2012 American Institute of Physics. [http://dx.doi.org/10.1063/1.4746748]
\end{abstract}

\section{INTRODUCTION}

The dynamic behavior of fluids in the bulk is characterized by transport properties such as the diffusion coefficient. In simple liquids, they are governed by the molecular interactions which can be effectively treated as pair potentials. In complex fluids, such as water, the dynamics of the systems is governed by network-forming interactions. The potentials involved are in general highly anisotropic and the transport properties tend to exhibit unusual behaviors.

Under confinement, even normal liquids have an unusual behavior, very different from the physical properties observed in bulk. The competition between surface effects and the confinement can induce a dramatic change in the transport properties of the fluid inside the channel. ${ }^{1-5}$

Bulk water is anomalous in many of its characteristics. The maximum in water's density is a well-known anomaly but there are many others. The self-diffusion coefficient at fixed temperature for a normal liquid decreases under compression, while in liquid water, it increases with the increase of pressure. In bulk water, this is due to the hydrogen bonds that are created and destroyed making particles to move from one neighbor to another neighbor.

Notwithstanding its molecular simplicity, water is quite hard to be modeled. The reason behind this difficulty is the presence of the hydrogen bonds, a non symmetric charge distribution and polarizability of the molecule that are density and temperature dependents. Consequently, there are more than 25 (bulk) water models for computational simulationempirical potentials - in which each of them gives a different dipole moment, dielectric and self-diffusion constants,

\footnotetext{
a)Electronic mail: bordin@if.ufrgs.br.

b)Electronic mail: oliveira@iceb.ufop.br.

c) Electronic mail: diehl@ufpel.edu.br.

d) Electronic mail: marcia.barbosa@ufrgs.br.
}

average configurational energy, density maximum, and expansion coefficient. More specifically, the maximum of density is found experimentally to be at $T=4{ }^{\circ} \mathrm{C}$ (for pressure $P$ $=1 \mathrm{~atm}$ ) while such models give values ranging from $-45^{\circ} \mathrm{C}$ (simple particle charge (SPC) model) up to $25^{\circ} \mathrm{C}$ (polarizable five-site (POL5/TZ) model). The transferable intermolecular potential with 5 points (TIP5P) water model was built to match the $4{ }^{\circ} \mathrm{C}$ experimental result, but it fails in many other aspects. ${ }^{6}$ Despite these limitations, these models have been used to understand the transport properties and phase transitions of confined water. ${ }^{7-15}$ The results give a qualitative comparison with experiments without providing a complete understanding of the origin of the anomalies. ${ }^{16}$ The majority of the molecular water models are conceived focusing on accurately describe the hydrogen bonds and charge distributions since many of the water uncommon properties are believed to come from its highly directional interactions. Examples are solvation and properties, which depend on polarization. On the other hand, the literature have many examples in which systems with the absence of anisotropic interactions may still present some of the water features. ${ }^{17-28}$ Some of its anomalous behavior may come from purely volumetric effects, which particularly is our focus in this work.

For confined systems, where water molecules interact in nanoscale distances, first principles simulations would be the appropriated tool for numerical comparison with experimental data. This procedure, however, has limitations. Even for confined water systems, in which the sizes involved are much smaller than that ones found in bulk cases, thousands of atoms are necessary for attacking typical problems along with millions of simulation steps. In this sense, it turns out that in the majority of cases, ab initio techniques become impracticable for dealing with such computational demanding systems.

Given the limitations of the full water models and the computational costs of the $a b$ initio simulations, classical 
effective empirical potentials became the simplest framework to understand the physics behind the anomalies of bulk water. From the desire of constructing a simple two-body potential capable of describing the anomalous behavior of bulk water, a number of models have been developed. ${ }^{17-28}$ Despite their simplicity, such models had successfully reproduced the thermodynamic, dynamic, and structural anomalous behavior present in bulk liquid water. They also predict the existence of a second critical point hypothesized by Poole and collaborators. ${ }^{29}$ This suggests that some of the unusual properties observed in water can be quite universal and possibly present in other systems.

In the case of confined water, a number of attempts have been made to understand its thermodynamic and dynamic properties. For the confinement media, nanotubes have been widely used for mimicking water confined into live organisms and as building blocks for technological applications, as desalination of water. ${ }^{30}$ Also, nanotubes can be used for drug delivery since they resemble biological ion channels. ${ }^{31}$ In addition, confinement in nanopores and nanotubes has also been used to avoid spontaneous water crystallization below the melting point in an attempt to observe its hypothetical second critical point. ${ }^{32-36}$ Simulations employing some of the discussed molecular models for water, namely SPC/E, TIP4PEW, and ST2, confined in nanoscale channels, exhibit two complementary effects: the melting temperature of the fluid at the center of the channel decreases and water crystallizes at the channel surface. ${ }^{37-41}$ In addition to these thermodynamic properties, the mobility properties of confined water also exhibit an unusual behavior. Experiments show an enhancement of the mobility orders of magnitude higher than what is predicted by the flow theories. ${ }^{42,43}$ Simulations $^{44-46}$ show an increase in the enhancement rate below a certain threshold radius. Similarly, the self-diffusion coefficient, $D$, obtained through molecular dynamic simulations for atomistic models, below a certain radius increases with decreasing radius. ${ }^{38,47-49}$

Besides the thermodynamic and dynamic unusual properties of confined water, the structure also presents an interesting behavior. The water structure inside larger nanotubes exhibits a layered structure, while in narrow nanotubes, a single file is observed. The layered water molecules can be found in a spiral-like chain, ${ }^{32}$ in a hexagonal structure for $(6,6)$ carbon nanotubes (CNT), ${ }^{38}$ a octagonal water-shell structure for a $(9,9)$ CNT, or a octagonal water-shell structure with a central water chain for a $(10,10) \mathrm{CNT}^{50,51}$ and others different structures. $^{41}$

The presence of layering effects is also controversial. Molecular dynamics (MD) simulations results by Wang et $a l .{ }^{52}$ do not show any obvious ordered water structure for $(9,9)$ or $(10,10)$ CNTs, unlike the works of Koles et al. ${ }^{50,51}$ This difference in findings can be caused by the influence of the water model used and corresponding Lennard-Jones parameters. ${ }^{52}$ The molecular structure of water confined in nanotubes and the diffusion can be very different depending on the chosen water model to perform the simulations. ${ }^{53}$ This difference arises as a consequence of the fact that the water models used in classical all-atoms MD simulations, like SPC/E, TIP3P, TIP4P, etc., are parametrized for bulk simulations, and reproduce only few aspects of real water. So, these models may show errors to describe the correct water behavior under strong confinement, like inside nanotubes.

The behavior of the diffusion coefficient with the nanotube radius is still under debate. Some simulations show a monotonical decrease of $D$ with decreasing nanotube radius ${ }^{54-56}$ while other simulations indicate the presence of a minimum. ${ }^{38,47-49}$ It has been suggested that the length of the nanotube and the length of the simulation would be responsible for the different results ${ }^{3,57,58}$ and that the friction should also play a relevant role. ${ }^{59}$

Our core-softened model introduced to study bulk system does not have any directionality and therefore it is not water. However, it does exhibit the density, the diffusion, and the response functions anomalies observed in water. This suggests that some of the anomalous properties that are attributed to the directionality of water can be found in spherical symmetry systems. Similarly, here we also propose that as the nanotube radius is decreased, the minimum in the diffusion coefficient observed for water can also be found in spherical symmetric systems.

In order to check our hypothesis, we model a water-like fluid into a nanotube using a core-softened potential. We test if this model is capable to capture the increase in the diffusion coefficient when the channel radius decreases. Next, we verify if the layering and the structure formed inside the channel, observed in some classical models for confined liquid water, has an universal feature or if it is just a consequence of the specific confining surface and model details.

The paper is organized as follows. The nanotube, waterlike fluid model, and the simulational details are presented in Sec. II. Our results are discussed in Sec. III, and the conclusions and summary are presented in Sec. IV.

\section{THE MODEL AND THE SIMULATION DETAILS}

\section{A. The model}

The water-like fluid is modeled as point particles with effective diameter $\sigma$ and mass $m$, interacting through the three dimensional core-softened potential ${ }^{17,18}$ (see Fig. 1)

$$
\frac{U\left(r_{i j}\right)}{\epsilon}=4\left[\left(\frac{\sigma}{r_{i j}}\right)^{12}-\left(\frac{\sigma}{r_{i j}}\right)^{6}\right]+u_{0} \exp \left[-\frac{1}{c^{2}}\left(\frac{r-r_{0}}{\sigma}\right)^{2}\right] .
$$

The first term in Eq. (1) is the standard Lennard-Jones (LJ) 12-6 potential ${ }^{60}$ and the second term is a Gaussian centered in $r_{0} / \sigma$, with depth $u_{0} \epsilon$ and width $c \sigma$. For $u_{0}=5.0, c=1.0$, and $r_{0} / \sigma=0.7$, this equation represents a two length scale potential, with one scale at $r_{i j} \approx 1.2 \sigma$, when the force has a local minimum, and the other scale at $r_{i j} \approx 2 \sigma$, where the fraction of imaginary modes has a local minimum. ${ }^{20}$ de Oliveira et al. ${ }^{17,18}$ obtained the pressure-temperature phase diagram of this system and showed that it exhibits thermodynamic, dynamic, and structural anomalies similar to the anomalies present in water. ${ }^{61,62}$

Here, we study the dynamic behavior of this water-like model confined in a nanotube connected to two reservoirs. 


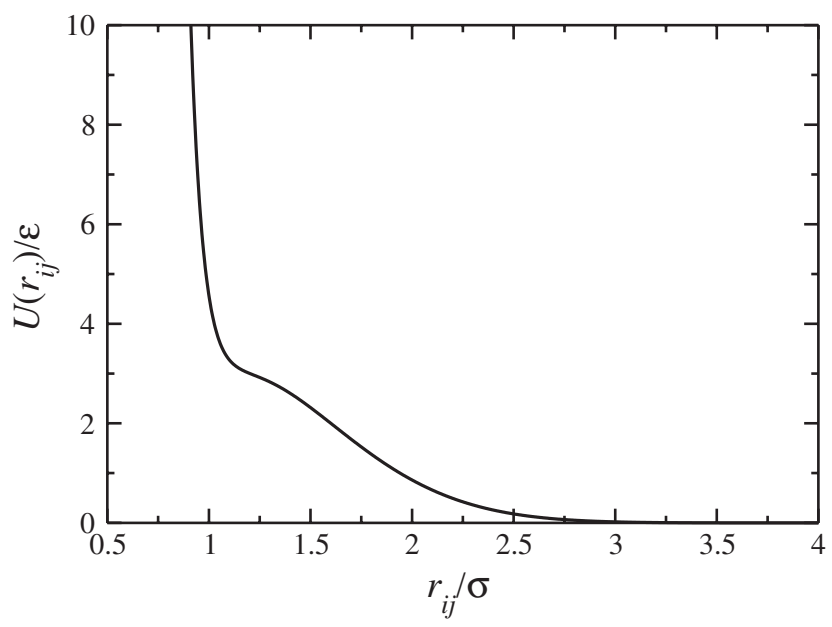

FIG. 1. Interaction potential between water-like particles.

The nanotube-reservoir setup is illustrated in Fig. 2. The simulation box is a parallelepiped with dimensions $L_{x} \times L_{y}$ $\times L_{z}$.

Two fluctuating walls, A in left and B in right, are placed in the limits of the $x$-direction of the simulation box. The walls are allowed to move in order to maintain the pressure constant in the reservoirs. The sizes $L_{y}$ and of $L_{z}$ depend on the effective nanotube radius, $a$, and they are defined by $L_{y}=L_{z}=L$ $=2 a+6 \sigma$. The initial size $L_{x}$ is given by $L_{x}=6 L_{c}$, where $L_{c}$ is the tube length. The nanotube structure was constructed as a wrapped hexagonal lattice sheet of point particles whose diameter is $\sigma_{\mathrm{NT}}=\sigma$. The nanotube interacts with the waterlike particles through the Weeks-Chandler-Andersen (WCA) potential $^{60}$ given by

$$
U_{i j}^{\mathrm{WCA}}(r)= \begin{cases}U_{\mathrm{LJ}}(r)-U_{\mathrm{LJ}}\left(r_{c}\right), & r \leq r_{c}, \\ 0, & r>r_{c},\end{cases}
$$

where $U_{\mathrm{LJ}}(r)$ is the standard LJ potential. The cutoff distance for this interaction is $r_{c}=2^{1 / 6} \sigma_{i j}$, where $\sigma_{i j}=\left(\sigma_{i}+\sigma_{j}\right) / 2$ is the center-to-center distance between the fluid particle $i$ and the nanotube particle $j$.

\section{B. The simulation details}

The properties of the system were evaluated with simulations at constant number of particles, pressure, and temperature ( $N p T$ ensemble). The Andersen thermostat, ${ }^{63}$ with col-

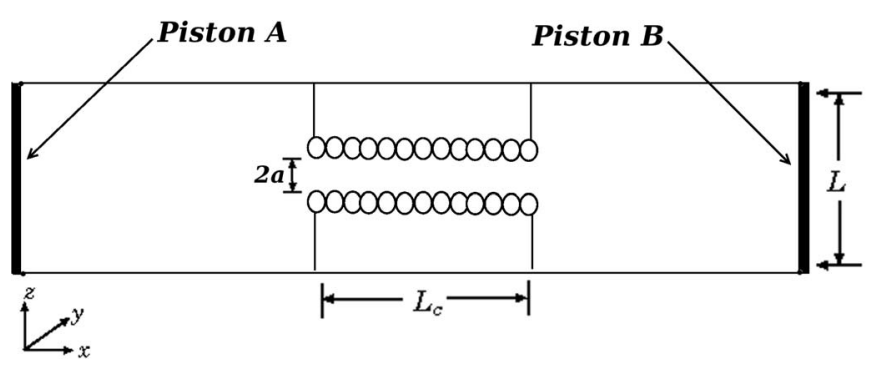

FIG. 2. Schematic depiction of the simulation box with the nanotube, reservoirs, and fluctuating walls. The cylindrical channel in the center has a radius $a$ and length $L_{c}$. The reservoirs have height $L$. lision frequency $v \delta t=0.01$, was used to maintain the temperature fixed. The pressure in both reservoirs was fixed using the Lupowski and van Smol method of fluctuating confining walls. ${ }^{64,65}$ These fluctuating walls act like pistons in the system where a constant force controls the pressure in the $x$ direction. This lead us to rewrite the resulting force in a waterlike particle as

$$
\vec{F}_{R}=-\vec{\nabla} U_{i j}+\vec{F}_{i w A}\left(\vec{r}_{i A}\right)+\vec{F}_{i w B}\left(\vec{r}_{i B}\right),
$$

where $\vec{F}_{i w j}$ indicates the interaction between the particle $i$ and the piston $j$. These forces were calculated from a WCA potential similar to Eq. (2), however considering the $x$-projection of the distance between one particle in the bulk and the piston position.

The equation of motion for the pistons are

$$
m_{w} \vec{a}_{A}=p S_{w} \vec{n}_{A}-\sum_{i=1}^{N} \vec{F}_{i w A}\left(\vec{r}_{i A}\right)
$$

and

$$
m_{w} \vec{a}_{B}=p S_{w} \vec{n}_{B}-\sum_{i=1}^{N} \vec{F}_{i w B}\left(\vec{r}_{i B}\right),
$$

where $m_{w}$ is the piston mass, $p$ the desired pressure in the system, $S_{w}$ is the piston area, and $\vec{n}_{A}$ is a unitary vector in positive $x$-direction, while $\vec{n}_{B}$ is a negative unitary vector. Both pistons (A and $\mathrm{B}$ ) have mass $m_{w}=m=1$, width $\sigma_{w}^{x}=\sigma$, and area equal to $S_{w}=L^{2}$. The Andersen thermostat is also applied to the pistons to ensure the temperature control. The values of pressure and temperature were chosen avoiding the density anomaly and the solid state regions. ${ }^{17,18}$

For simplicity, we assume that the nanotube atoms are fixed (i.e., not time integrated) during the simulation. The reduced quantities are defined as usual,

$a^{*} \equiv \frac{a}{\sigma}, \quad \rho^{*} \equiv \rho \sigma^{3}, \quad t^{*} \equiv t\left(\frac{\epsilon}{m \sigma^{2}}\right)^{1 / 2}, \quad$ and $T^{*} \equiv \frac{k_{B} T}{\epsilon}$,

for the channel radius, density of particles, time and temperature, respectively, and

$$
p^{*} \equiv \frac{p \sigma^{3}}{\epsilon} \quad \text { and } \quad D^{*} \equiv \frac{D(m / \epsilon)^{1 / 2}}{\sigma},
$$

for the pressure and diffusion coefficient, respectively. Periodic boundary conditions were applied in the $y$ and $z$ directions. The equations of motion for the particles of the fluid were integrated using the velocity Verlet algorithm, with a time step $\delta t^{*}=0.005$. The fluid-fluid interaction, Eq. (1), has a cutoff radius $r_{\text {cut }}^{*}=3.5$. The nanotube radius was varied from $a^{*}=1.25$ to $a^{*}=10.0$, and the number of fluid particles in the simulations varies from 500 to 3500 . The number of particles were chosen considering that the nanotube would be filled with the fluid and that we would have in the reservoirs, the same properties evaluated in previous $N V T$ simulation for the non-confined case. ${ }^{17,18}$ For all values of radius, the nanotube length was defined as $L_{c}^{*}=20$.

Five independent runs were performed to evaluate the properties of the fluid inside the nanotube. For each simulation run, half of fluid particles was initially placed into each 


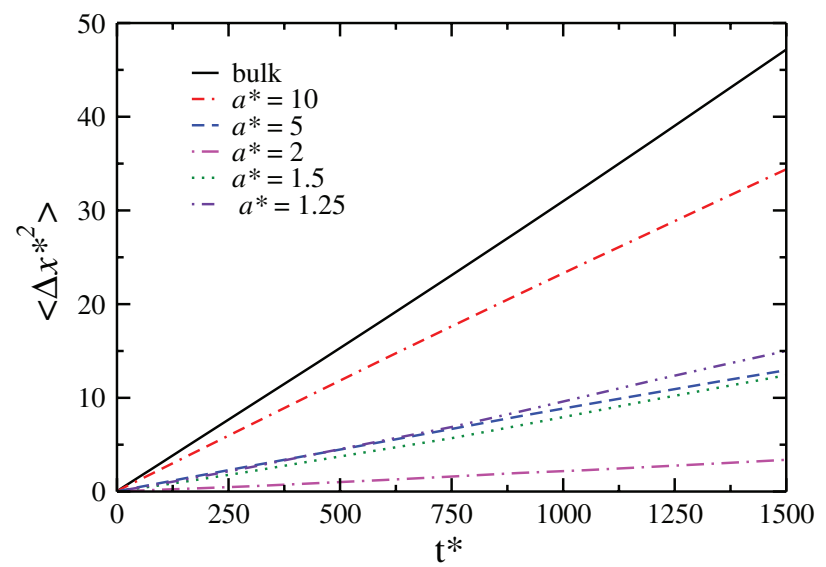

FIG. 3. Axial mean square displacement versus time for channel radius $a^{*}=1.25,1.5,2.0,5.0$, and 10.0 .

reservoir. We performed $5 \times 10^{5}$ steps to equilibrate the system followed by $5 \times 10^{6}$ steps for the results production stage. The equilibration time was taken in order to ensure that the nanotube became filled with water-like particles as well as the pistons reached the equilibrium position for a given pressure.

For calculating the axial diffusion coefficient, $D_{x}$, we computed the axial mean square displacement (MSD), namely,

$$
\left\langle\left[x(t)-x\left(t_{0}\right)\right]^{2}\right\rangle=\left\langle\Delta x(t)^{2}\right\rangle=2 D t^{\alpha},
$$

where $x\left(t_{0}\right)$ and $x(t)$ denote the axial coordinate of the confined water-like molecule at a time $t_{0}$ and a later time $t$, respectively. The diffusion coefficient $D_{x}$ is then obtained from

$$
D_{x}=\lim _{t \rightarrow \infty} \frac{\left\langle\Delta x(t)^{2}\right\rangle}{2 t^{\alpha}} .
$$

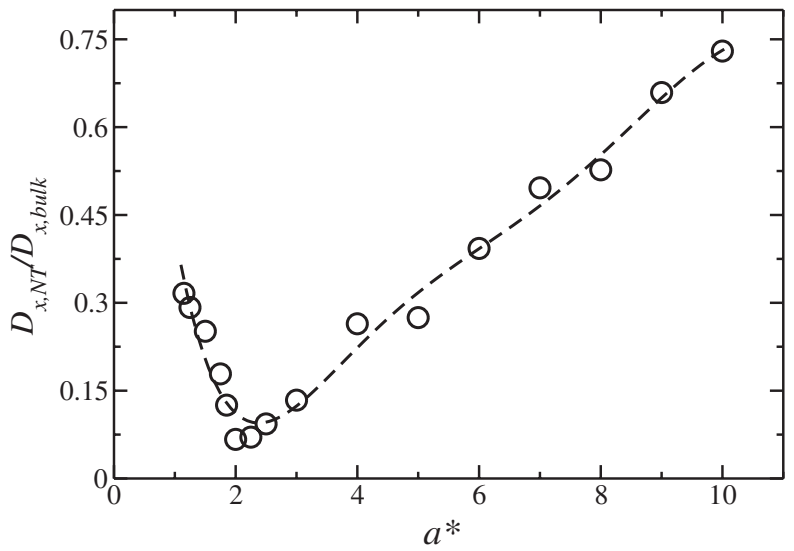

FIG. 4. Diffusion coefficient inside nanotube, $D_{x, \mathrm{NT}}$, in units of nonconfined diffusion, $D_{x}$, bulk, for different nanotube radius. The error bars are smaller than the data point. The dotted line is a guide to the eye.

Depending on the scaling law between $\Delta x^{2}$ and $t$ in the limit $t \rightarrow \infty$, different diffusion mechanisms can be identified: $\alpha$ $=0.5$ identifies a single file regime, ${ }^{48} \alpha=1.0$ stands for a Fickian diffusion, whereas $\alpha=2.0$ refers to a ballistic diffusion. $^{3,41,48,49,58}$

\section{RESULTS AND DISCUSSION}

First, we checked which is the diffusive regime of our system for different channel radius. Figure 3 illustrates the axial mean square displacement versus time for channel radius $a^{*}=1.25,1.5,2.0,5.0$, and 10.0 at $T^{*}=0.25$ and $p^{*}=0.7$. For simple LJ confined fluids for very narrow channels, a single-file diffusion regime is found $(\alpha=0.5){ }^{3,4}$ Instead, in our model, Fickian diffusion was observed for all channel radius, $(\alpha=1.0)$. This result is in agreement with the diffusion coefficient at the center of nanotubes observed for
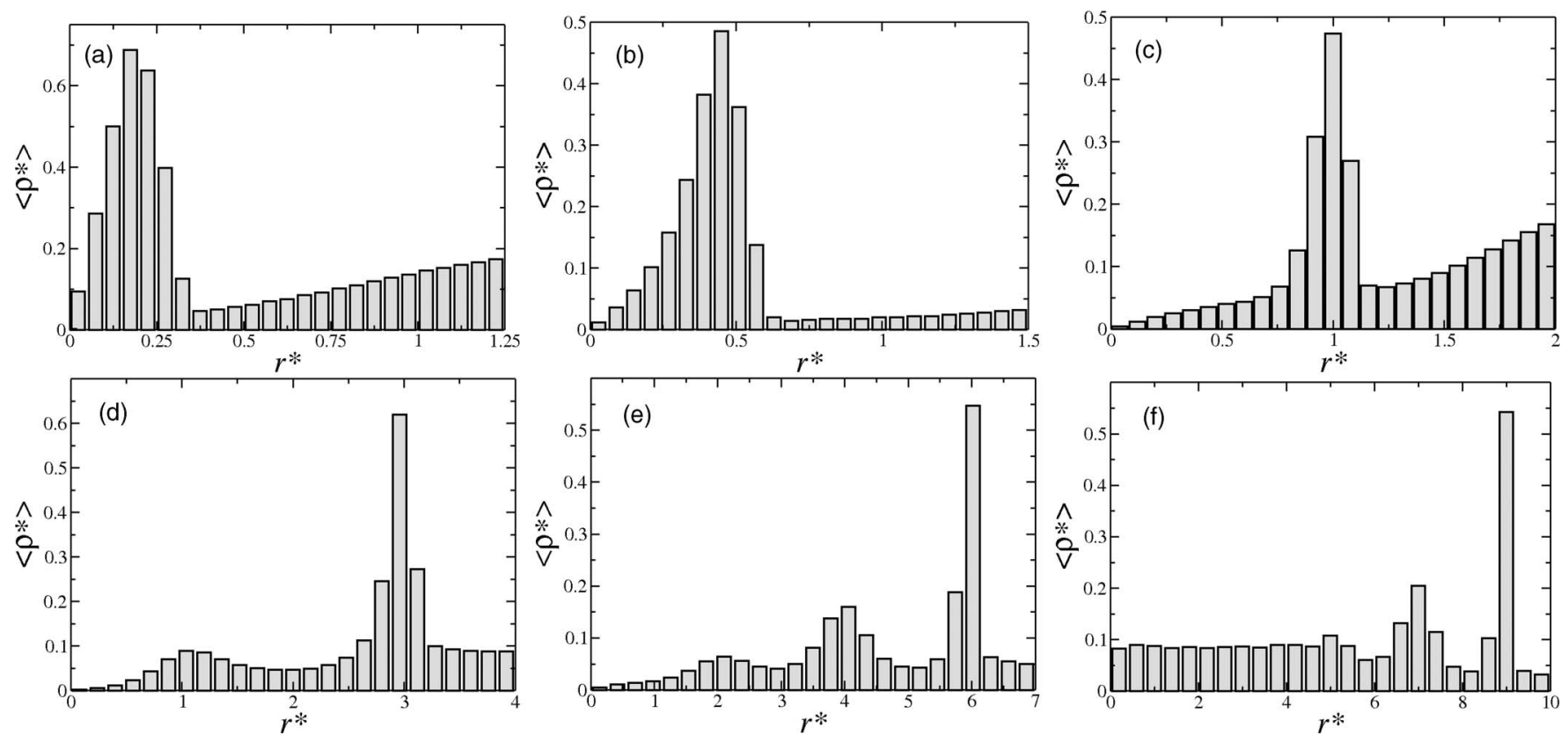

FIG. 5. Radial density profile for different values of radius: (a) $a^{*}=1.25$, (b) $a^{*}=1.5$ (c) $a^{*}=2.0$, (d) $a^{*}=4.0$, (e) $a^{*}=7.0$, and (f) $a^{*}=10.0$. 


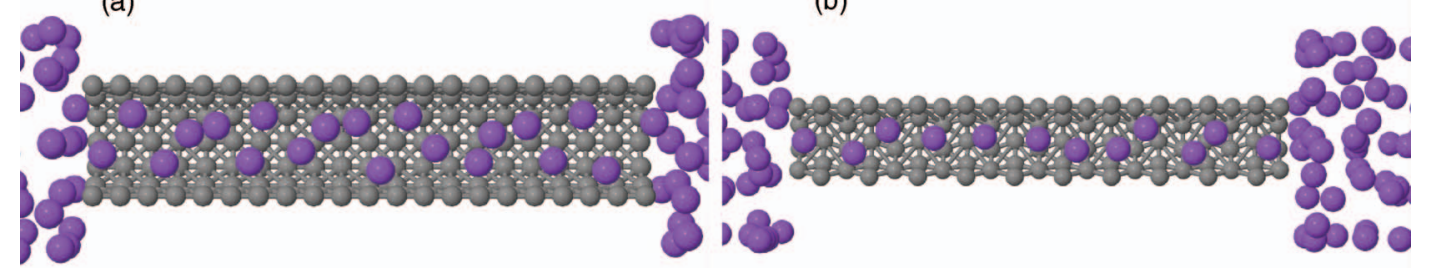

FIG. 6. Snapshots of the system for (a) $a^{*}=2.0$ and (b) $a^{*}=1.5$.

water models SPC/EP, ${ }^{3,38}$ SPC,${ }^{54}$ TIP3P, ${ }^{53}$ and TIP4P. ${ }^{49}$ In the case of water, the Fickian diffusion has been interpreted as a combination of highly coordinated like in a ballistic mode and a monodimensional configuration like in a single-line diffusion. ${ }^{41,58}$ The presence of a highly coordinated structure was observed in water in very narrow nanotubes ${ }^{48}$ and between plates, ${ }^{66}$ confirming the interpretation of Mukherjee et $a .^{58}$ In our case, the particles also combine a strongly correlated motion with a single-line diffusion. As we are going to see in detail below, the molecules are arranged in shells. The motion inside each cell is correlated and slow while the motion between shells is faster. The compromise between these two mechanisms leads to a Fickian diffusion.

It is important to stress that the major difference between a simple LJ fluid and our core-softened model is the presence of two-scales in the potential (absent in the LJ fluid). This enforces our conjecture that two scales in the interatomic potential play an important role in the appearance of water-like features.

Next, we tested if the diffusion through the channel obeys the mean-field-like Knudsen equation, i.e., if the diffusion coefficient is proportional to the channel radius. Figure 4 illustrates the diffusion coefficient, $D$, versus channel radius, $a^{*}$, for fixed $T^{*}=0.25$ and $p^{*}=0.7$. We see from this figure that a critical channel radius $a_{c}^{*}$ exists where the derivative of the $D(a)$ curve is zero. For $a^{*}>a_{c}^{*}=2.0$, the diffusion coefficient presents the expected behavior of increasing with $a^{*}$. For large channel radius, the growth is linear as predicted by the Knudsen equation. For $a^{*}<2.0$, on the other hand, we observed that $D$ decreases with increasing $a^{*}$, which cannot be explained by the Knudsen mean-field approach. At $a^{*}=a_{c}^{*}=2.0$, particles are virtually immobilized, i.e., $D \approx 0$.

Studies for SPC/E, ${ }^{38,48,55}$ TIP4P-EW, ${ }^{47,49}$ and SPC (Refs. 54 and 56) show that the diffusion coefficient increases with the channel radius, $a$. Simulations for SPC/E (Refs. 38 and 48) and TIP4P-EW (Refs. 47 and 49) also show the decrease of the diffusion coefficient with the increase of the channel radius for $a<a_{c}$. This anomalous region is captured by our model and it is not observed in our simulations for LJ confined fluids (details not shown here for simplicity).

For SPC/E and TIP4P-EW potentials used for confined water, the number of neighbors and the number of hydrogen atoms differ from those numbers in the bulk phase. In these models, the different slope in the $D(a)$ function, i.e., positive for $a>a_{c}$ and negative for $a<a_{c}$, are attributed to a competition between two effects: the confinement and the nanoscale surface. For the $a>a_{c}$ case, $D$ decreases for decreasing $a$ be- cause of the confinement. This is not hard to understand since decreasing $a$ allows less space for particles to move. ${ }^{38,47-49}$ Increasing confinement leads to surface effects becoming more important. In the water case, hydrogen bonds from the surface are depleted and molecules become more mobile. This would explain why $D$ increases for decreasing $a$ below $a_{c} .^{38,47-49}$ The behavior of $D$ in water, therefore, can be explained by minimizing the free energy. Water molecules have a gain in rotational entropy when they are come inside a narrow carbon channel. ${ }^{67,68}$ This gain in entropy compensates the lost in enthalpy due to the reduction of number of hydrogen bonds. ${ }^{12,48}$

Interestingly though our system does not have hydrogen bonds, thus it is not subject to the competition between hydrogen bonds depletion and diminishing space available for particles to move. Therefore, what would be the mechanism behind our non-monotonic curve $D(a)$ shown in Fig. 4?

The behavior of the diffusion coefficient in our model can be understood by two complementary ways. First, by examining the density profile inside the nanotube. The density distribution is computed in cylindrical coordinates, $r^{2}=y^{2}+$ $z^{2}$, where $r=0$ is the center of the channel. Figure 5 illustrates the radial density profile versus $r^{*}$ for the channel radii $a^{*}=1.25,1.5,2.0,4.0,7.0$, and 10.0. In all analyzed cases, layering is observed. Axial layers are also observed in simulations for the SPC/E and TIP4P-EW models for water confined in nanotubes. ${ }^{5,38,46,55,58}$ In the last cases, the presence of layering is attributed to the hydrogen bonds and surface effects. In our model, the presence of layering comes as a result

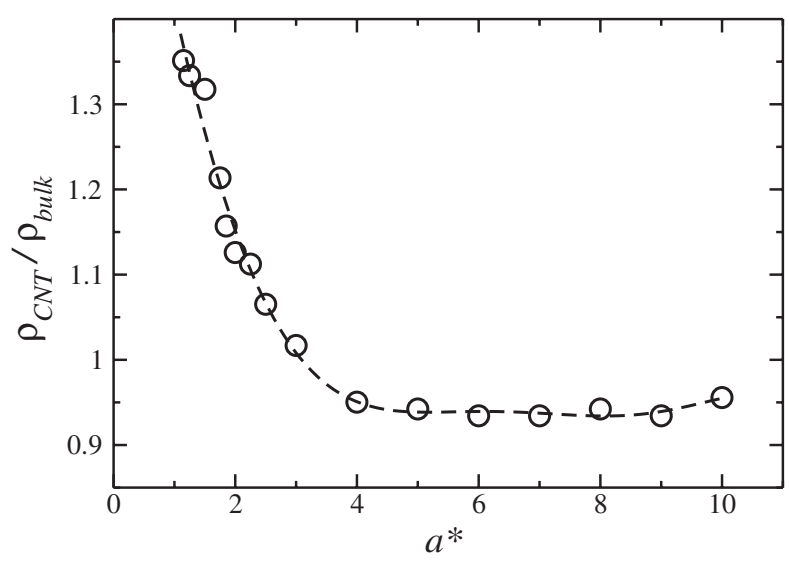

FIG. 7. Total density inside the nanotube as function of the tube radius for $T^{*}=0.25$ and $p^{*}=0.7$. The error bars are smaller than the data point. The line is a guide to the eye. 

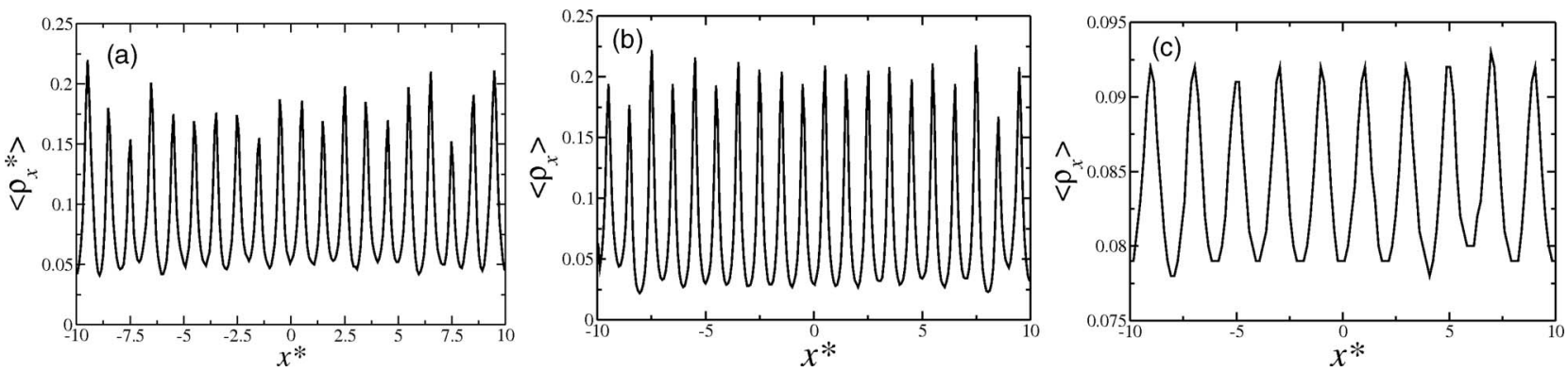

FIG. 8. Axial density profile for a nanotube with radius (a) $a^{*}=1.5$, (b) $a^{*}=2.0$, and (c) $a^{*}=7.0$.

of the competition between particle-particle and particle-wall interactions. The potential illustrated in Fig. 1 favors particles to be at least at $r_{p p}^{*}=2.0$ apart, while the hydrophobic walls push particles away to a distance of at least $r_{p w}^{*}=2^{1 / 6}$. Consequently, for $a^{*}=1.25,1.5,2.0,4.0$, and 7.0, a number of layers equal to $1,1,2,4$, and 6 are formed. For $a^{*} \geq 10$, a continuous distribution emerge. For $a^{*}>2.0$, the system form layers arranged in distances that minimize the potential energy. This indicates that for larger diameters, the enthalpic contribution for the free energy dominates over the entropic contribution. For $a^{*}<2.0$, there is only one layer of particles. Since the particle-wall interaction is purely repulsive, particles advance over the wall repulsive region moving "free" in the radial direction and entropy increases and the mobility rises. For $a^{*}<2.0$, the entropic contribution dominates over the enthalpic contribution.

The decrease in the diffusion coefficient as the channel radius is decreased for $a^{*}>a_{c}^{*}=2.0$ is associated with the layers formation and particularly with the correlation between particles in different layers that try to move without changing the layer to layer distance. As the number of layers increase for $a^{*}>a_{c}^{*}=2.0$, fluctuations allow particles to move faster. At $a^{*}=a_{c}^{*}=2.0$, the diffusion reaches a minimum and the system assumes a crystal-like configuration as illustrated in Figure 8. Therefore, the confinement leads the fluid to a solidlike state, even at values of temperature and pressure far from solid state phase. . $^{58,41,51,55}$

In order to check what happens as the channel radius is decreased further, snapshots of the system for $a^{*}=2.0$ and $a^{*}$ $=1.5$ are shown in Fig. 6. For $a^{*}=2.0$, particles form two layers while for $a^{*}=1.5$, a single layer is observed. The correlations that immobilizes particles at the two layers structure disappear as the single layer is formed and particles can diffuse faster for $a^{*}<2.0$ by moving from the close packing (at $\left.r^{*}=1.0\right)$ to the minimum of the fraction of imaginary modes (at $r^{*}=2.0$ ).

Besides the layering in the radial direction, particles also change their structure in the axial direction. Figure 7 shows the total density inside the nanotube as a function of the radius. For $a^{*}<4.0$, the density of the confined system increases with the decrease of $a^{*}$. This result is qualitatively the same observed for the recent simulations of SPC/E model of water in a nanotube-reservoirs system. ${ }^{46}$ This can be explained by a change in the axial distance between particles. Figure 8 for $a^{*}=1.5$ illustrates that for small radius, the pref- erential axial distance is the shoulder scale, $x^{*}=1.0$, while in the bulk and for larger radius, the preferential axial distance is $x^{*} \approx 2.0$.

\section{CONCLUSION}

This paper explores the connection between the surface interaction, confinement, and the presence of two length scales in the diffusion of a fluid in narrow channels. The water-like fluid was modeled using a spherically symmetric two length potential, and the confining channel is modeled as hard spheres. Our system shows an enhancement of the diffusion coefficient with the decrease of the channel radius for a channel radius below a certain critical value. This effect arises from the competition between the confinement that accommodates particles at the lower energy length scale, and the surface interaction that pushes particles away from the surface generating correlated layers. For wider channels, the layers are accommodated minimizing the potential energy forming an organized structure. For narrow channels, the particles form a single layer that move more freely advancing over the wall repulsion and therefore increasing the diffusion. The mechanism is quite similar to the one proposed for water.

In addition, we found that below a certain channel radius, the density inside the channel is larger than the bulk density. This is explained on basis of the length scales competition and it is in agreement with simulations. Our results indicate that the presence of minimum in the diffusion coefficient with the decrease of the channel radius is not a property solely due to directional systems such as water, but can also be seen in spherical symmetric systems or in system in which directionality would not play a relevant role.

\section{ACKNOWLEDGMENTS}

This work was partially supported by the CNPq, CAPES, FAPERGS, and INCT-FCx.

${ }^{1}$ Z. G. Mao and S. B. Sinnott, J. Phys. Chem. B 104, 4618 (2000).

${ }^{2}$ Y.-C. Liu, J. D. Moore, T. J. Roussel, and K. E. Gubbins, Phys. Chem. Chem. Phys. 12, $6632(2010)$.

${ }^{3}$ A. Striolo, Nanoletters 6, 633 (2006).

${ }^{4}$ J. Pikunic and K. E. Gubbins, Eur. Phys. J. E 12, 35 (2003).

${ }^{5}$ G. E. Karniadakis, A. Beskok, and N. R. Aluru, Microflows and Nanoflows-Fundamentals and Simulation (Springer, New York, 2005).

${ }^{6} \mathrm{M}$. Chaplin, Water Models, see http://www.lsbu.ac.uk/water/models.html. 
${ }^{7}$ M. Rovere and P. Gallo, Eur. Phys. J. E 12, 77 (2003).

${ }^{8}$ I. Brovchenko, A. Geiger, A. Oleinikova, and D. Paschek, Eur. Phys. J. E 12, 69 (2003).

${ }^{9}$ N. Giovambattista, P. J. Rossky, and P. G. Debenedetti, Phys. Rev. Lett. 102, 050603 (2009).

${ }^{10}$ S. Han, M. Y. Choi, P. Kumar, and H. E. Stanley, Nat. Phys. 6, 685 (2010).

${ }^{11}$ T. G. Lombardo, N. Giovambattista, and P. G. Debenedetti, Faraday Discuss. 141, 359 (2009).

${ }^{12}$ F. de los Santos and G. Franzese, J. Phys. Chem. B 115, 14311 (2011).

${ }^{13}$ P. Gallo, M. Rovere, and S.-H. Chen, J. Phys.: Condens. Matter 24, 064109 (2012).

${ }^{14}$ E. G. Strekalova, M. G. Mazza, H. E. Stanley, and G. Franzese, J. Phys.: Condens. Matter 24, 064111 (2012).

${ }^{15}$ M. Melillo, F. Zhu, M. A. Snyder, and J. Mittal, J. Phys. Chem. Lett. 2, 2978 (2011).

${ }^{16}$ F. Mallamace, C. Corsaro, P. Baglioni, E. Fratini, and S.-H. Chen, J. Phys.: Condens. Matter 24, 064103 (2012).

${ }^{17}$ A. B. de Oliveira, P. A. Netz, T. Colla, and M. C. Barbosa, J. Chem. Phys. 124, 084505 (2006).

${ }^{18}$ A. B. de Oliveira, P. A. Netz, T. Colla, and M. C. Barbosa, J. Chem. Phys. 125, 124503 (2006).

${ }^{19}$ J. N. da Silva, E. Salcedo, A. B. de Oliveira, and M. C. Barbosa, J. Chem. Phys. 133, 244506 (2010).

${ }^{20}$ A. B. de Oliveira, E. Salcedo, C. Chakravarty, and M. C. Barbosa, J. Chem. Phys. 132, 234509 (2010).

${ }^{21}$ N. M. Barraz, Jr., E. Salcedo, and M. C. Barbosa, J. Chem. Phys. 131, 094504 (2009).

${ }^{22}$ A. B. de Oliveira, G. Franzese, P. Netz, and M. C. Barbosa, J. Chem. Phys. 128, 064901 (2008).

${ }^{23}$ A. B. de Oliveira, P. Netz, and M. C. Barbosa, Eur. Phys. J. B 64, 481 (2008).

${ }^{24}$ A. B. de Oliveira, P. Netz, and M. C. Barbosa, Europhys. Lett. 85, 36001 (2009).

${ }^{25}$ J. R. Errington, T. M. Truskett, and J. Mittal, J. Chem. Phys. 125, 244502 (2006).

${ }^{26}$ J. Mittal, J. R. Errington, and T. M. Truskett, J. Phys. Chem. B 110, 18147 (2006).

${ }^{27}$ W. P. Krekelberg, J. Mittal, V. Ganesan, and T. M. Truskett, Phys. Rev. E 77, 041201 (2008)

${ }^{28}$ L. Xu, S. V. Buldyrev, N. Giovambattista, C. A. Angell, and H. E. Stanley, J. Chem. Phys. 130, 054505 (2009).

${ }^{29}$ P. H. Poole, F. Sciortino, U. Essmann, and H. E. Stanley, Nature (London) 360, 324 (1992).

${ }^{30}$ M. Elimelech and W. A. Philip, Science 333, 712 (2011).

${ }^{31}$ T. A. Hilder, D. Gordon, and S. H. Chung, Nanomedicine 7, 702 (2011).

${ }^{32}$ L. Liu, S. H. Chen, A. Faraone, C. W. Yen, and C. Y. Mou, Phys. Rev. Lett. 95, 117802 (2005).

${ }^{33}$ F. Mallamace, C. Branca, C. Corsaro, N. Leone, J. Spooren, H. E. Stanley, and S. H. Chen, J. Phys. Chem. B 114, 1870 (2010).

${ }^{34}$ S. H. Chen, F. Mallamace, C. Y. Mou, M. Broccio, C. Corsaro, A. Farone, and L. Liu, Proc. Natl. Acad. Sci. U.S.A. 103, 12974 (2006).

${ }^{35}$ O. Mishima and H. E. Stanley, Nature 392, 164 (1998).
${ }^{36}$ H. E. Stanley, S. V. Buldyrev, P. Kumar, F. Mallamace, M. G. Mazza, K. Stokely, L. Xu, and G. Franzese, J. Non-Cryst. Solids 357, 629 (2011).

${ }^{37}$ L. D. Gelb, K. E. Gubbins, R. Radhakrishnan, and M. S. Bartkowiak, Rep. Prog. Phys. 62, 1573 (1999).

${ }^{38}$ R. J. Mash, S. Joseph, and N. R. Aluru, Nanoletters 3, 589 (2003).

${ }^{39}$ M. C. Gordillo and J. Martí, Chem. Phys. Lett. 329, 341 (2000).

${ }^{40}$ H. Kyakuno, K. Matsuda, H. Yahiro, Y. Inammi, T. Fukukoa, Y. Miyata, K. Yanagi, H. Kataura, T. Saito, M. Tumura, and S. Iijima, Chem. Phys. Lett. 134, 244501 (2011).

${ }^{41}$ A. Alexiadis and S. Kassinos, Chem. Rev. 108, 5014 (2008).

${ }^{42}$ J. K. Holt, H. G. Park, Y. M. Wang, M. Stadermann, A. B. Artyukhin, C. P. Grigoropulos, A. Noy, and O. Bakajin, Science 312, 1034 (2006).

${ }^{43}$ M. Majumder, N. Chopra, R. Andrews, and B. J. Hinds, Nature (London) 438, 44 (2005)

${ }^{44}$ J. A. Thomas and A. J. H. McGaughey, Nanoletters 8, 2788 (2008).

${ }^{45}$ J. A. Thomas and A. J. H. McGaughey, Phys. Rev. Lett. 102, 4502 (2009).

${ }^{46}$ X. Qin, Q. Yuan, Y. Zhao, S. Xie, and Z. Liu, Nanoletters 11, 2173 (2011).

${ }^{47}$ H. Ye, H. Zhang, Y. Zheng, and Z. Zhang, Microfluid. Nanofluid. 11, 1359 (2011).

${ }^{48}$ A. B. Farimani and N. R. Aluru, J. Phys. Chem. B 115, 12145 (2011).

${ }^{49}$ Y. Zheng, H. Ye, Z. Zhang, and H. Zhang, Phys. Chem. Chem. Phys. 14, 964 (2012).

${ }^{50}$ A. I. Kolesnikov, J. M. Zanotti, C. K. Loong, and P. Thiygarajan, Phys. Rev. Lett. 93, 035503 (2004).

${ }^{51}$ A. I. Kolesnikov, C. K. Long, N. R. de Souza, C. J. Burnham, and A. P. Moravsky, Physica B 385, 272 (2006).

${ }^{52}$ J. Wang, Y. Zhu, J. Zhou, and X. H. Lu, Phys. Chem. Chem. Phys. 6, 829 (2004)

${ }^{53}$ A. Alexiadis and S. Kassinos, Chem. Eng. Sci. 63, 2793 (2008).

${ }^{54}$ Y. C. Liu, J. W. Shen, K. E. Gubbins, J. D. Moore, T. Wu, and Q. Wang, Phys. Rev. B 77, 125438 (2008).

${ }^{55}$ T. Nanok, N. Artrith, P. Pantu, P. A. Bopp, and J. Limtrakul, J. Phys. Chem. A 113, 2103 (2009).

${ }^{56}$ Y. Liu, Q. Wang, and L. Zhang, J. Chem. Phys. 123, 234701 (2005).

${ }^{57}$ J. Su and H. Guo, J. Chem. Phys. 134, 244513 (2011).

${ }^{58}$ B. Mukherjee, P. K. Maiti, C. Dasgupta, and A. K. Sood, J. Chem. Phys. 126, 124704 (2007).

${ }^{59}$ K. Falk, F. Sedlmeier, L. Joly, R. R. Netz, and L. Bocquet, Nanoletters 10, 4067 (2010).

${ }^{60} \mathrm{P}$. Allen and D. J. Tildesley, Computer Simulation of Liquids (Oxford University Press, Oxford, 1987).

${ }^{61}$ G. S. Kell, J. Chem. Eng. Data 12, 66 (1967).

${ }^{62}$ C. A. Angell, E. D. Finch, and P. Bach, J. Chem. Phys. 65, 3063 (1976).

${ }^{63}$ H. C. Andersen, J. Chem. Phys. 72, 2384 (1980).

${ }^{64}$ M. Lupowski and F. van Smol, J. Chem. Phys. 93, 737 (1990).

${ }^{65}$ A. P. Thompson and G. S. Heffelfinger, J. Chem. Phys. 110, 10693 (1999).

${ }^{66}$ F. de los Santos and G. Frazese, Phys. Rev. E 85, 010602 (2012).

${ }^{67}$ H. Kumar, B. Mukherjee, S.-T. Lin, and C. Dasgupta, J. Chem. Phys. 134, 124105 (2011)

${ }^{68}$ T. A. Pascal, W. A. Goddard, and Y. Jung, Proc. Natl. Acad. Sci. U.S.A. 108, 11794 (2011) 